
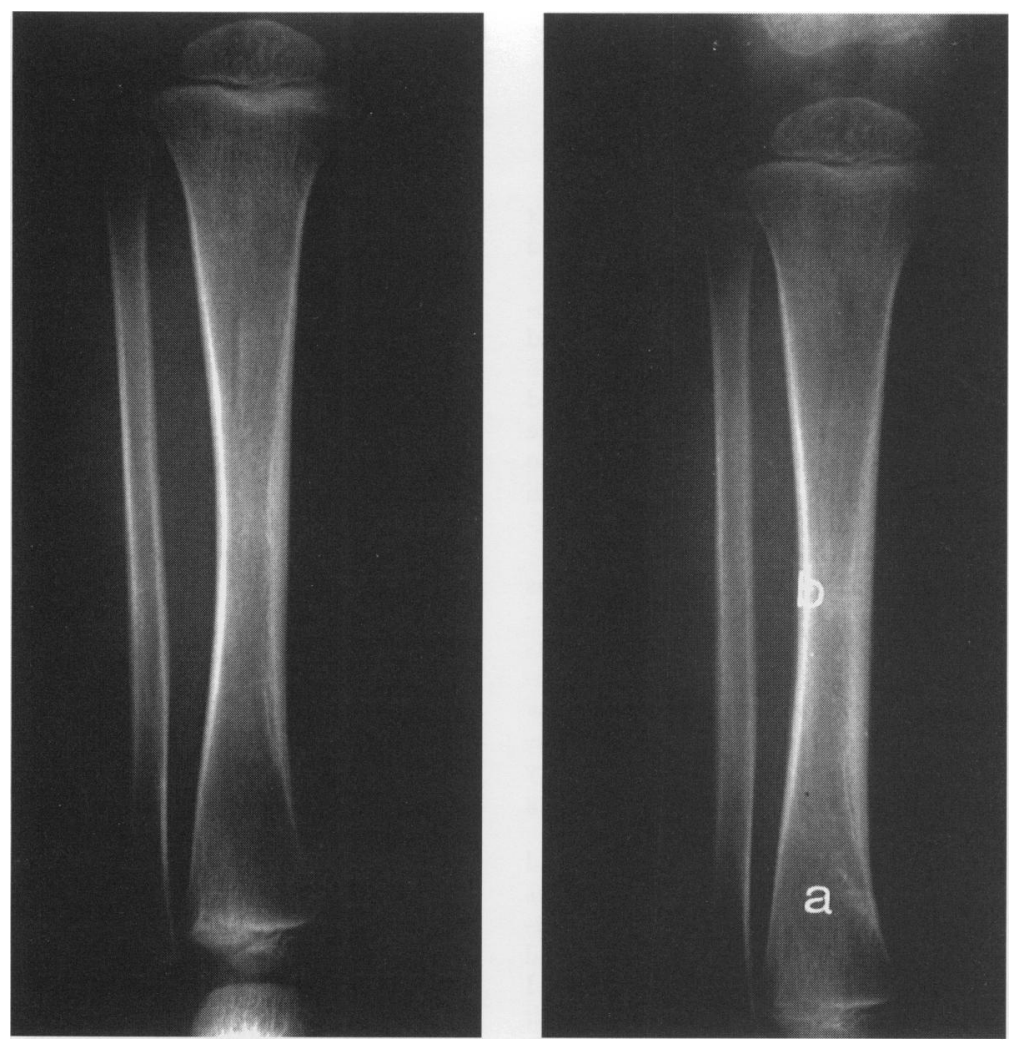

Left: $A 2$ year old with limping and tenderness over the distal tibia. No abnormality is seen. Right: $A$ fracture line [spinal pattern (a)] is now shown over the lower third of the tibia with periosteal reaction along the tibial shaft (b).

Children per year and we see 70 toddler's fractures (as defined above) each year.

In our experience not all these children require immobilisation of the limb in a plaster of Paris. We reserve such treatment for children when sleep or daily activities are disturbed by pain. In this age group the extra weight of an above knee cast for a parent to carry, combined with the hygiene challenge in a group either wearing nappies or being toilet trained, outweighs any advantage to the child except for analgesic purpose as stated above.

Any discussion on fractures in young children should include reference to nonaccidental injury as the cause and we feel this should not have been omitted from this paper.

$$
\begin{array}{r}
\text { J CLANCY } \\
\text { L PIETERSE } \\
\text { P ROBERTSON } \\
\text { D MCGRATH } \\
\text { T F BEATTIE } \\
\text { Royal Hospital for Sick Children, } \\
\text { Edinburgh }
\end{array}
$$

1 Shravat BP, Harrop SN, Kane TP. Toddler's fracture. F Accid Emerg Med 1996;13:59-61.

\section{Fast tracking patients with a proximal}

\section{femoral fracture}

EDITOR, - In relation to the paper by Ryan et $a l^{1}$ on "fast tracking" patients with proximal femoral fractures, we have operated a fast track system for proximal femoral fractures on and off for several years. In our system, uncomplicated cases, defined by a protocol, are received nurse requested radiology, the $x$ rays, when returned, are briefly assessed by an A\&E doctor, and the patient is then admitted directly to the ward and the orthopaedic $\mathrm{SHO}$ informed. If there is time in $A \& E$ and the department is not too busy, we will also cannulate the patient and do relevant blood tests and an ECG, but not invariably. This system seems to work well. However, a recent audit showed disappointing results in that there were still unacceptable delays for some patients. The chief reasons for the delay were long periods spent in the $x$ ray department and problems with unfamiliar orthopaedic junior staff refusing to accept patients on such a basis, and insisting on assessing them in the A\&E department. This has now been addressed and the system will be reaudited soon.

B J FINLAYSON

Norfolk and Norwich Health Care NHS Trust

1 Ryan J, Ghani $M$, Staniforth P, Bryant G, Edwards S. "Fast tracking" patients with a proximal femoral fracture. F Accid Emerg Med

\section{BOOK REVIEWS}

\section{Fundamentals of Emergency Radiology.}

By Philip Wiest and Paul Roth. (Pp 175;

£27.95.) Philadelphia: W B Saunders, 1996. ISBN 0721651828.

This is a superbly illustrated text on emergency radiology and is aimed primarily at physicians and medical students involved in the emergency department. As its title indicates, it is not a definitive text on any of the areas covered but concentrates on practical aspects of selecting the most appropriate imaging techniques. This is facilitated by a number of excellent flow charts. These have been applied in each of the chapters, which include imaging of the head and spine, chest, abdomen urinary tract, pelvis, and orthopaedics. 1996;13:108-110.
A number of useful "pearls" are enumerated at the end of each chapter, emphasising the most important points for the practising clinician. As indicated, the image quality is of the highest standard and unlike many texts the radiological abnormalities are easily visualised and well annotated.

There are a number of transatlantic idiosyncrasies which do not translate well to the British system. This may in part relate to the availability of high tech equipment. Other basic differences include their routine use of PA and lateral chest films for patients with chest pain, where a PA chest $x$ ray in the first instance is considered adequate in most UK centres.

These are, however, minor differences of opinion which do not detract from what is an excellent, compact, easily readable text. I would recommend this to anyone starting in an emergency department; it may even be of use to trainee radiologists as it covers the basic groundwork of emergency radiology.

P M HUGHES

Plymouth

Emergency Medical Therapy. By T F

Mengert, M S Eisenberg, and M K Copass

(Pp 992; f33.00.) Philadelphia: W B

Saunders, 1996. ISBN 0721651623.

This chunky little book wants to be a pocket manual for all known medical specialties. It's a big ambition for a relatively small book, but achieves it in the main. The problem is that if you are going to ruin your white coat pockets, it needs to be with a book that covers everything.

The majority of adult medical emergencies are covered, and the therapeutic options are presented in an easy to read and logical way. Some of the first line drugs are unfamiliar, or would not be first choice in this country, but there is lots of really practical advice on diagnostics as well as suggested starting doses and predicted response levels. Internal headings for chapters follow the same pattern throughout, and include pearls and pitfalls as well as suggestions on disposition of the patient. There are some helpful flow diagrams dealing, for example, with headaches or CNS symptoms in HIV infected patients. These are mixed with some more weighty and rather indigestible tables. The first chapter on general care of the emergency department patient could easily form the basis of the SHO induction talk.

The recurring theme is early assessment/ resuscitation, followed by a more leisurely discourse on possible causes and remedies. Many of the chapters put me in mind of practice exam answers in their eagerness to follow a pattern and they would therefore be useful for revision. It also lends itself to a source of reference for emergency nurses in this country.

There are a few missing medical topics that I would have enjoyed reading the solution toperhaps confusional states, and the care of the frail elderly. Additionally, although hand infections are addressed in great detail, little else is offered for the care of the "walking wounded". This is a pity because, judging from the section on conscious sedation, local anaesthetic techniques and blocks as well as wound care would have been usefully discussed.

Overall, I like this book but wish it was either smaller and lighter, or, by extending its scope to surgery/trauma cases, it could be a 